\title{
Evaluating the roles of detailed endocardial structures on right ventricular haemodynamics by means of CFD simulations
}

\author{
Federica Sacco ${ }^{1,2}$ | Bruno Paun ${ }^{2}$ | Oriol Lehmkuhl ${ }^{1}$ | \\ Tinen L. lles $^{3}$ | Paul A. laizzo ${ }^{3}$ | Guillaume Houzeaux ${ }^{1}$ \\ Mariano Vázquez ${ }^{1,4}$ \\ Constantine Butakoff $^{2}$ ।

\section{Jazmin Aguado-Sierra ${ }^{1 *}$}

${ }^{1}$ Computer Applications in Science \& Engineering (CASE), Barcelona

Supercomputing Center, Barcelona, Spain

${ }^{2}$ PhySense, ETIC, Universitat Pompeu

Fabra, Barcelona, Spain

${ }^{3}$ Visible Heart Laboratory, Department of Surgery, University of Minnesota,

Minneapolis, United States

${ }^{4}$ IIIA - CSIC, Bellaterra, Barcelona, Spain

\section{Correspondence}

Jazmin Aguado-Sierra, Barcelona

Supercomputing Center, 08034, Barcelona,

Spain

Email: jazmin.aguado@bsc.es

Funding information

J. A.S., M. V., Barcelona Supercomputing

Center; CompBioMed H2O2O-EU.1.4.1.3

( ${ }^{\circ}$ 675451). F. S., Barcelona

Supercomputing Center, Universitat

Pompeu Fabra; Severo Ochoa

(n`SEV-2015-0493-16-4). C. B., Universitat

Pompeu Fabra; Fundació La Marató de TV3

(n`20154031). T.L. I., P.A. I., University of

Minnesota; Institute of Engineering in

Medicine, Lillehei Heart Institute.
Computational modelling plays an important role in right ventricular (RV) haemodynamic analysis. However, current approaches employ smoothed ventricular anatomies. The aim of this study is to characterise RV haemodynamics including detailed endocardial structures like trabeculae, moderator band and papillary muscles (PMs). Four paired detailed and smoothed RV endocardium models (two male and two female) were reconstructed from ex-vivo human hearts high-resolution magnetic resonance images (MRI). Detailed models include structures with $\geq 1 \mathrm{~mm}^{2}$ cross-sectional area. Haemodynamic characterisation was done by computational fluid dynamics (CFD) simulations with steady and transient inflows, using high performance computing (HPC). The differences between the flows in smoothed and detailed models were assessed using Q-criterion for vorticity quantification, the pressure drop between inlet and outlet, and the wall shear stress (WSS). Results demonstrated that de-

Abbreviations: RV, Right Ventricle; PMs, Papillary Muscles; MRI, Magnetic Resonance Imaging; CFD, Computational Fluid Dynamics; HPC, High Performance Computing; WSS, Wall Shear Stress; LV: Left Ventricle; FSI, Fluid Structure Interaction; PBS, Phosphate Buffered Saline; BSC, Barcelona Supercomputing Center; FEM, Finite Element Method; FE, Finite Element; PDFs: Probability Density Functions 
tailed endocardial structures increase the degree of intraventricular pressure drop, decrease the WSS and disrupt the dominant vortex creating secondary small vortices. Increasingly turbulent blood flow was observed in the detailed RVs. Female RVs were less trabeculated and presented lower pressure drops than the males. In conclusion, neglecting endocardial structures in RV haemodynamic models may lead to inaccurate conclusions about the pressures, stresses, and blood flow behaviour in the cavity.

\section{KEYWORDS}

haemodynamics, right ventricle, CFD, HPC, endocardial structures

\section{1 | INTRODUCTION}

The RV's primary function is to receive systemic venous return and then pump this de-oxygenated blood to the lungs via the pulmonary arteries. Specifically, during diastole, when the RV relaxes, it begins to fill with blood that flows from the atrium to the ventricle and dilates; this flow is through the tricuspid valve and final filling occurs with right atrial contraction (atrial kick). Subsequently, during systole, the RV contracts and pumps the blood into the pulmonary trunk and then to left and right pulmonary arteries; hence delivering blood to the lungs for oxygenation. The right and left heart pumps function in series. Therefore, $\mathrm{RV}$ haemodynamics plays an critical role in the overall heart function [18, 34] and associated valve or contractile malfunctions may lead to pulmonary hypertension, RV myocardial infarction and heart failure. Today, although rapidly improving, in vivo clinical imaging tools for cardiac haemodynamic assessments are somewhat limited in their accessibilities, resolutions, and/or accuracies; therefore, giving computational modelling an exciting role for predicting modulations in blood flows. Yet to date, most of RV computational models and/or experimental studies, have been quite limited as compared to the ones that have been reported for the left ventricle (LV): most likely because of considered higher importances of the LV for the perfusing the heart and body. Further, it should be noted that additional challenges exsist in the assessments of RV functions and haemodynamicsis its shape, which is far from elliptical [9], making it difficult to find representative views using stadardised imaging modalities. As a result, most described computational modelling of the RV haemodynamics, consider this chamber with a smoothed endocardium. In a report by Pasipoularides et al., [20] they analysed the diastolic filling vortices in seven normal and overloaded smoothed canine RV models. They described that the blood flow extended from the tricuspid orifice to the RV endocardial surface with an "expanding fan-like pattern" during peak E wave. Subsequently, during RV filling, they also observed the formation of large-scale vortices, moving from the ventricular walls towards the ventricular base, suggesting that these vortices may preserve energy and help with the ejection phase. In another study, Pasipoularides and colleagues [21] observed that there were contributions by local and convective acceleration gradients to RV diastolic instantaneous intra-ventricular pressures; they observed such using functional imaging via 3D real-time echocardiography. These also reported that early RV diastolic intra-ventricular pressure gradients were very small, due to an underlying balance between convective deceleration and local acceleration components during the early filling upstroke. Vruddhula et al. [35] studied the 3D blood flow pattern within a simplified, smoothed RV model: they observed the creation of a vortex ring, which breaks into smaller structures during the end of the E-wave generating a 
swirling flow. Subsequently, smoothed patient-specific bi-ventricular models were used by Yang et al. [37] to investigate $\mathrm{RV}$ functions and to analyse RV-LV interactions using fluid-structure interactions (FSI). Their haemodynamic simulations were run on simple smoothed ventricular models and were conducted under varying pressure and material conditions. They employed patches of diverse geometries and stiffnesses applied on the RV, so to analyse their relative effects on overall ventricular functions. Their simulations predicted that the blood flows, the stress and the strain distributions within the RV, were related to its morphologies, material properties and a given patient's blood flow and pressure conditions. Moreover, Su et al. [28] used right ventricular CFDs to analyse modulations in blood flows within a normal $\mathrm{RV}$ and a pathological one affected by pulmonary arterial hypertension. RV smoothed geometry was reconstructed manually from MRI data throughout a cardiac cycle. From normal subject simulation, the authors observed a clockwise swirling flow inside the ventricular cavity during early diastole, while the apical flow was smooth and slow during the whole cardiac cycle. Pulmonary arterial hypertension led to slower trans-pulmonary flow and no swirl of the blood within the RV cavity was observed. Moreover, in normal patient the vortex structures penetrated deeper as compared to the pathological case. Further, Wiputra et al. [34] analysed the CFD in three smoothed patient-specific fetal RV models. These authors identified the generation of two independent vortex rings, which merged during diastole. Moreover, these vortices generated velocities that were tangent to the ventricular wall and led to high WSS in these regions during diastole; whereas the role of the vortex rings in systolic WSS was minimal.

Despite the abundance of papers considering smoothed-endocardium RV, the latter is characterised by a very trabeculated endocardium (more than LV), two PMs, and the moderator band, which is a conduction band that extends from the ventricular septum to the base of the anterior PM and would significantly affect the blood flow pattern and vortex formation. Several studies demonstrated how considering detailed endocardial structures in CFD affects the LV haemodynamics [15, 17, 32]. In these works, the authors analysed blood flow patterns within complex ventricular cavities, considering trabeculae and PMs. While the amount of trabeculations in the ventricular models of Lantz et al. [17] and Vedula et al. [32] was relatively minimal, the model of Kulp et al. [15] was highly trabeculated. Importantly, both Vedula et al. [32] and Lantz et al. [17] findings suggested that the detailed LV endocardial structures disrupted the vortices, generating secondary vortices that filled the empty spaces between the endocardial trabecular architecture, just to be expelled out of these spaces during systole.

In our study, we characterised the geometrical effects of trabeculae, PMs and the moderator band on the blood flows inside two female and two male human RV models using CFD simulations. Each RV model presented both a smoothed (representative of the state of art) and detailed endocardial cavity. The detailed RV models included endocardial structures with a minimum cross-sectional area of $1 \mathrm{~mm}^{2}$. With both the smoothed and detailed RV models, we initially ran constant inflow CFD simulations. Subsequently, we ran transient inflow simulations by generating a synthetic, physiological E-A wave. In this way, we were able to both characterise the pure impact of the trabeculations on the blood flow and quantify the effect of neglecting these structures in haemodynamic modelling with the analysis of intra-ventricular pressure drop, geometrical markers, WSS and blood flow vorticity inside the ventricular chambers. Moreover, we could investigate possible gender differences and/or similarities and analyse blood flow turbulence.

\section{2 | MATERIALS AND METHODS}

\section{1 | Data}

Four RV models were reconstructed from high resolution MRI data of ex-vivo perfusion fixed human hearts, obtained from the Visible Heart ${ }^{\circledR}$ Laboratory at the University of Minnesota. The uses of these heart specimens in research have received appropriate approval from both the University of Minnesota's Institution Review Board and LifeSource 
Research Committee (Minnesota's non-profit organ and tissue procurement donation organisation). These heart specimens were recovered from organ donors, whose hearts were unsuitable for transplantation. Both written and informed consents were obtained from the donors families, which follow the wishes of the donor. The four hearts were then fixed with $10 \%$ formalin in a solution of phosphate buffered saline (PBS) for a minimum of 24 hours under a pressure of $40-50 \mathrm{mmHg}$ and then stored in $10 \%$ formalin. Subsequently, the images of the fixed hearts were acquired via a 3T Siemens scanner with $0.44 \times 0.44 \mathrm{~mm}$ in-plane resolution and slice thickness of 1 to $1.7 \mathrm{~mm}$. The hearts were fixed at constant pressure to preserve end diastolic volume. The four datasets and the corresponding medical history are shown in Fig. 1 and Table 1 of the supplementary materials.

\section{2 | Model construction}

Image segmentation was done using the software Fiji [25], with the maximum entropy-based thresholding algorithm [23]. Subsequently, the reconstructions of the detailed endocardial surfaces of the four RV models were done using marching cubes algorithm in Seg3D [6]. The images had an excellent contrasting gray scales, which allowed thresholding to produce highly detailed endocardial models. The smoothed geometries were created from the detailed ones by a manual selection of the smoothed endocardial envelopes for each image slice. Autodesk Meshmixer [26] sculpting software was then used to adjust the smoothed endocardial envelopes so to correctly enclose the detailed profiles.

Within this study it was considered important to include open tricuspid valves for two main reasons: the valve directs the jet of blood within the geometry, which determines regions of blood impinging inside the cavity; and secondly, the blood flow recirculation behind the valve leaflets is an important feature in ventricular haemodynamics. The tricuspid valves were manually segmented from the images using Seg3D. Valve geometries were then merged with the segmented ventricles of both smoothed and detailed models. The valves were assumed to be rigid in this study.

Paraview [10] was used to attach 90 mmlong tubes at the inlet (tricuspid valve orifice) and at the outlet (pulmonary valve orifice) of every RV model to let the flow fully develop. The tubes were positioned approximately perpendicularly to the respective valves. The obtained meshes were uniformly remeshed with Remesh [1] and volumetric tetrahedral meshes were created using an isosurface-stuffing-based algorithm [16] with an in-house mesher developed at the Barcelona Supercomputing Center (BSC). These volumetric meshes had varying element sizes, from $2.8 \cdot 10^{-7} \mathrm{~mm}^{3}$ to $2.6 \cdot 10^{-2} \mathrm{~mm}^{3}$, with an average size of $7.6 \cdot 10^{-5} \mathrm{~mm}^{3}$. Zoomed wire-frame tetrahedral meshes images can be found in Fig. 2 of the electronic supplementary materials.

The anatomies of the four RV models, two females $\left(F_{1,2}\right)$ and two males $\left(M_{1,2}\right)$, are shown in Fig. 1, each one shown with its respective smoothed and detailed endocardium. The ventricular volume of each smoothed and detailed RV is reported in Table 1, along with the volume occupied by the trabeculations (percentage of trabeculae) in each case.

\section{3 | Haemodynamic simulations}

Blood flow at normal diastolic pressures were modelled using the Navier-Stokes equations for incompressible flows, considering Newtonian viscous stresses. Simulations were carried out on both MareNostrum 4 supercomputer and Archer, UK supercomputer, using BSC's in-house, parallel multi-physics, HPC solver Alya [11, 31] with a finite element method (FEM) based strategy described below.

The Navier-Stokes equations for a fluid domain $\Omega$ bounded by $\Gamma=\partial \Omega$ during the time interval $\left(t_{0}, t_{f}\right)$ consist in 

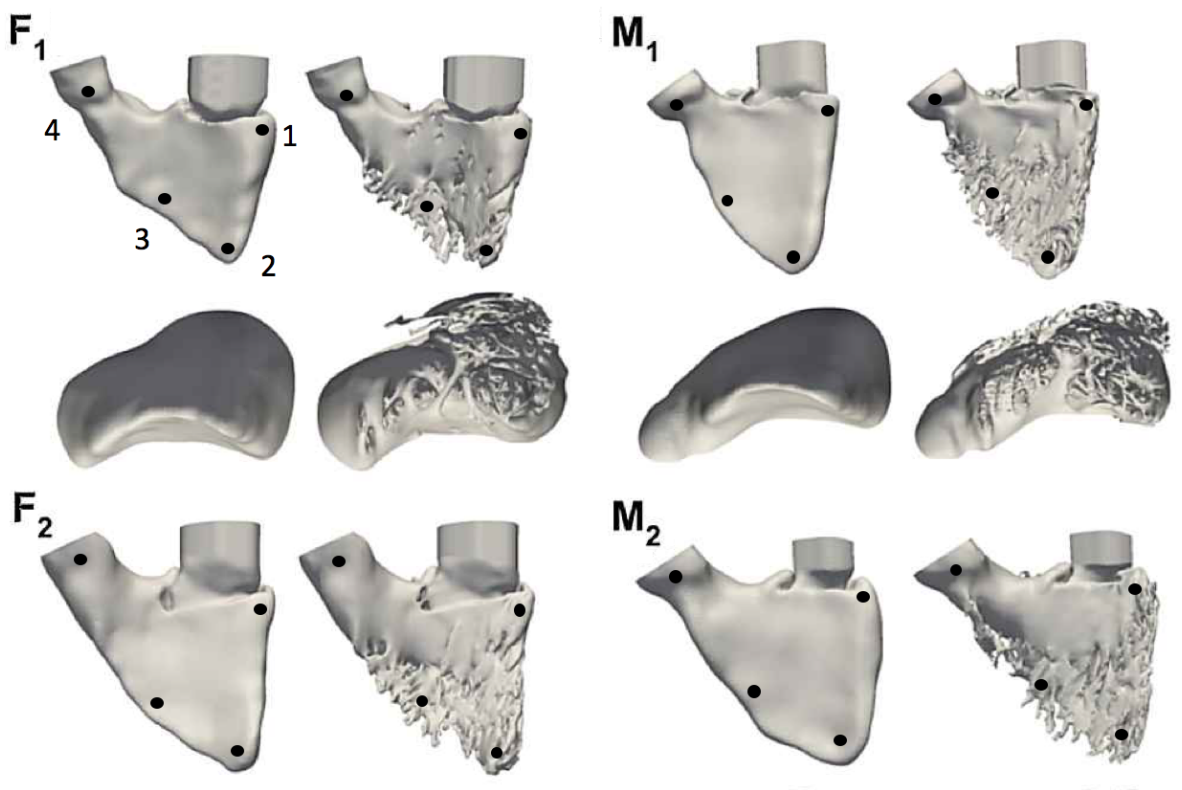

$\mathrm{M}_{2}$
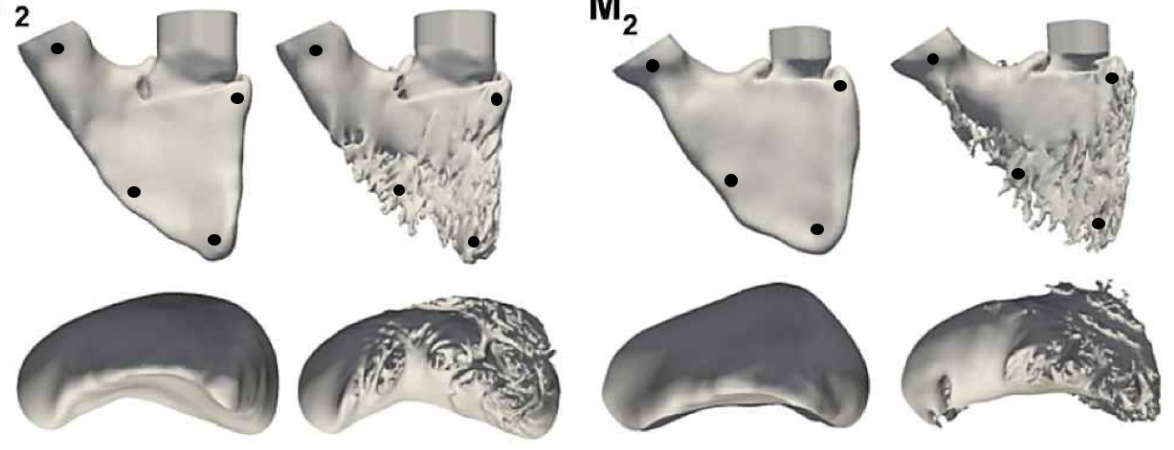

FIG URE 1 Female $\left(F_{1,2}\right)$ and male $\left(M_{1,2}\right)$ RV models with smoothed (left) and detailed (right) endocardial surfaces. The points indicated (1-4) represent the four points of interest that were used to record velocity signals and analyse their auto-correlation. Below each RV, the corresponding section of the ventricular cavity is shown.

finding a velocity $\mathbf{u}$ and a kinematic pressure $p$ such that

$$
\begin{aligned}
\partial_{t} \mathbf{u}+(\mathbf{u} \cdot \nabla) \mathbf{u}-2 v \nabla \cdot \mathbf{S}(\mathbf{u})+\nabla p-\mathbf{f}=0 & \text { in } \Omega \times\left(t_{0}, t_{f}\right), \\
\nabla \cdot \mathbf{u}=0 & \text { in } \Omega \times\left(t_{0}, t_{f}\right),
\end{aligned}
$$

where $v$ was the kinematic viscosity, $\mathbf{f}$ the vector of external body forces and $\mathbf{S}(\mathbf{u})$ was the rate-of-strain tensor.

In order to obtain the weak or variational formulation of the Navier-Stokes equations (eqns. (1) and (2)), we introduce the spaces of vector functions $\mathbf{V}_{D}=\mathbf{H}_{D}^{1}(\Omega), \mathbf{V}_{0}=\mathbf{H}_{0}^{1}(\Omega)$ and $Q=L^{2}(\Omega) / R$. $L^{2}(\Omega)$ was the space of squareintegrable functions, $H^{1}(\Omega)$ was a subspace of $L^{2}(\Omega)$ formed by functions whose derivatives also belong to $L^{2}(\Omega)$, $H_{D}^{1}(\Omega)$ was a subspace of $H^{1}(\Omega)$ that satisfies the Dirichlet boundary condition on $\Gamma, H_{0}^{1}(\Omega)$ is a subspace of $H^{1}(\Omega)$ whose functions were zero on $\Gamma$, and $\mathbf{H}_{D}^{1}(\Omega)$ and $\mathbf{H}_{0}^{1}(\Omega)$ were their vector counterparts in either a two- or threedimensional space. $(\cdot, \cdot)$ indicates the standard $L^{2}$ inner product. For the evolutionary case, $\mathbf{V}_{t} \equiv L^{2}\left(t_{0}, t_{f} ; \mathbf{V}_{D}\right)$ and $Q_{t} \equiv \mathcal{D}^{\prime}\left(t_{0}, t_{f} ; Q\right)$ were introduced, where $L^{p}\left(t_{0}, t_{f} ; X\right)$ is the space of time dependent functions in a normed space $X$ such that $\int_{t_{0}}^{t_{f}}\|f\|_{X}^{p} \mathrm{~d} t<\infty, 1 \leq p<\infty$ and $Q_{t}$ consists of mappings whose $Q$-norm was a distribution in time. The weak form of problem $(1,2)$ with the boundary conditions was then: Find $\mathbf{u} \in \mathbf{V}_{t}, p \in Q_{t}$ such that 


$$
\left(\partial_{t} \mathbf{u}, \mathbf{v}\right)+(\mathbf{u} \cdot \nabla \mathbf{u}, \mathbf{v})+2 v(\mathbf{S}(\mathbf{u}), \nabla \mathbf{v})-(p, \nabla \cdot \mathbf{v})+(q, \nabla \cdot \mathbf{u})-(\mathbf{f}, \mathbf{v})=0
$$

for all $(\mathbf{v}, q) \in \mathbf{V}_{0} \times Q$.

In addition, in the previous equations the convective form of the non-linear term

$$
N L_{\text {conv }}(\mathbf{u})=\mathbf{u} \cdot \nabla \mathbf{u}
$$

has been used, which is probably the most frequent choice in computational practice. Using eqn. (2), other forms of the non-linear term can be derived, which were equivalent at the continuous level but have different properties at the discrete level. In the following, we consider the energy, momentum and angular momentum conserving form recently proposed in [5]

$$
N L_{e m a c}(\mathbf{u})=2 \mathbf{S}(\mathbf{u}) \mathbf{u}+(\nabla \cdot \mathbf{u}) \mathbf{u}
$$

A non-incremental fractional-step method was used to stabilise pressures. This allows for the use of finite element pairs that do not satisfy the inf-sup conditions, such as equal order interpolation for the velocities and pressures used in this work. The set of equations was time integrated using an energy conserving Runge-Kutta explicit method recently proposed by Capuano et al. [3] combined with an eigenvalue based time-step estimator [29]. This methodology most recently proposed by Lehmkuhl et al. [19], follows the same principles of Verstappen et al. [33], generalised for unstructured finite volumes by Jofre et al. [13] and Trias et al. [30], but in a FEM framework. The present methodologies has been successfully validated and benchmarked against other popular CFD approaches and for the experimental data in the context of bioengineering flows reported by Koullapis et al. [14].

\subsection{1 | Boundary conditions}

CFD simulations were carried out by labelling boundaries to define the inlets at the tricuspid and the outlets at the pulmonary tubes respectively (an illustration of the labels positions can be found in Fig. 3 of the electronic supplementary materials). In all simulations, the boundary condition proposed by Bazilevs et al. [2] was imposed at the outlets to set a baseline outlet pressure at $1066.6 \mathrm{~Pa}(8 \mathrm{mmHg})$, approximately equal to the mean pulmonary artery pressure of a normal heart. This boundary condition adds stability to the formulation in presence of locally reversed flow through an outflow resistance boundary [2]. Along with this condition, a layer of high viscosity $(0.035 \mathrm{~kg} /(\mathrm{m} \cdot \mathrm{s}))$ is added at the outlet tube to further stabilise the blood flow. No-slip, rigid walls conditions were applied to both tubes and RV walls to characterise the geometry and isolate the pure effect of the presence of detailed endocardial structures on the blood flow. For the haemodynamic settings blood viscosity was equal to $0.0035 \mathrm{~kg} /(\mathrm{m} \cdot \mathrm{s})$ and density to $1060 \mathrm{~kg} / \mathrm{m}^{3}$. In our experiments, two sets of simulations were performed:

- Constant inflow:

A constant blood flow velocity of $0.38 \mathrm{~m} / \mathrm{s}$ (peak normal trans-tricuspid inflow velocity [8]) was imposed as inflow to each of the models. This was done to characterise the sole geometrical influence on the RV haemodynamics.

- E-A wave tricuspid valve inflow:

A synthetic E-A wave was generated by combining two cosine functions as in (6). $A$ was the wave maximum 
amplitude $[\mathrm{m} / \mathrm{s}], t_{0, E}, t_{0, A}$ and $t_{1, E}, t_{1, A}$ were the $\mathrm{E}$ and $\mathrm{A}$ waves initial and final times [s], respectively. $t_{p, E}$ and $t_{p, A}$ were the peak $\mathrm{E}$ and $\mathrm{A}$ waves corresponding times $[\mathrm{s}]$ and $t$ was the simulation time $[\mathrm{s}]$. The wave parameters represented the normal E-A waves of a healthy human being [8]. Their values and the corresponding wave are reported in Fig. 2(a).

$$
v(t)= \begin{cases}\frac{A_{E}}{2}\left(1+\cos \left(\frac{2 \pi\left(t-t_{p, E}\right)}{t_{1, E}-t_{0, E}}\right)\right) & t_{0, E} \leq t \leq t_{1, E} \\ 0 & t_{1, E}<t<t_{0, A} \\ \frac{A_{A}}{2}\left(1+\cos \left(\frac{2 \pi\left(t-t_{p, A}\right)}{t_{1, A}-t_{0, A}}\right)\right) & t_{0, A} \leq t \leq t_{1, A}\end{cases}
$$

The E wave represents the early, passive filling of the RV cavity at the opening of the tricuspid valve; while the A wave represents the late filling, which is the $\mathrm{RV}$ inflow given by the right atrial contraction. These two waves are separated by a short period of time, named diastasis, where velocity is zero.

\section{4 | Data analysis}

Three geometrical markers were used for results analysis:

Trabecular volume was calculated as the percentage change between the volume of the smoothed $\left(V_{s}\right)$ and the the detailed $\left(V_{d}\right)$ ventricular cavities: $\frac{\left(V_{s}-V_{d}\right)}{V_{s}} \cdot 100$.

The angle between inlet and outlet was defined as the angle between the normals to the two valvular planes (tricuspid and pulmonary respectively), as observed in Fig. 2(b).

The distance between inlet and outlet was calculated as the distance between the centres of the tricuspid and pulmonary valve rings, as observed in Fig. 2(b).

The blood flow was quantified using the following measurements:

Intra-ventricular pressure drop. In order to assess the intra-ventricular pressure drops, pressure histograms were calculated within volumes of $15 \mathrm{~mm}$ thick sections at the inlet and outlet tubes. The histograms of the pressure drops were computed by bootstrapping [7] and normalised to unit area under the curve. Bin width was calculated using the Freedman-Diaconis rule. As the pressure probability density functions (PDFs) were not Gaussian (see pressure distribution plots in Fig. 4-7 of the electronic supplementary materials), the intra-ventricular pressure drops of detailed and smoothed RVs $\left(\Delta P_{d, s}\right)$ were calculated as the differences of the inlet and outlet pressures modes $\left(P_{\text {in }}-P_{\text {out }}\right)$ for each geometry. The values of $\Delta P_{d, s}$ were then averaged over the last ten time steps of the constant inflow simulations.

Subsequently, the intra-ventricular pressure drop differences $\left(\Delta P_{\text {diff }}\right)$ were calculated as the differences between the detailed and smoothed pressure drops $\left(\Delta P_{d}-\Delta P_{s}\right)$.

WSS on the ventricular walls. The WSS was defined as:

$$
W S S=\left.\mu_{d}\left(\frac{\partial u}{\partial y}\right)\right|_{y=0}
$$

Where $\mu_{d}$ is the dynamic viscosity [Pa $\left.\cdot \mathrm{s}\right], u$ is the flow velocity component parallel to the wall [m/s] and $y$ is the distance to the wall [m]. WSS histograms were computed in constant inflow simulations and normalised to the area under the curves. The Freedman-Diaconis rule was used to select the bins width. WSS PDFs and maximum value were calculated for each smoothed and detailed RV cavity (see WSS distribution plots in Fig. 8-11 of the electronic supplementary materials). Given that the PDFs were upward skewed, the median and the mode of the WSS were 


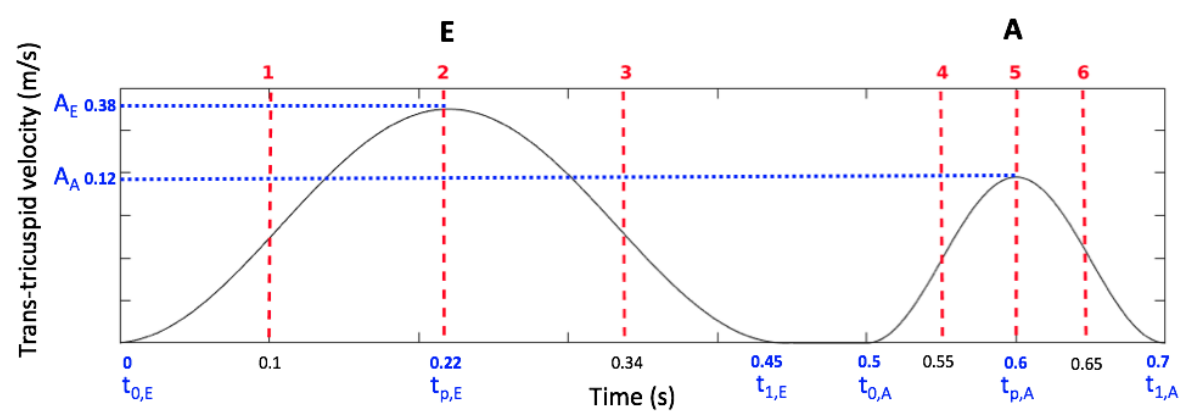

(a) Synthetic trans-tricuspid E-A wave input function.

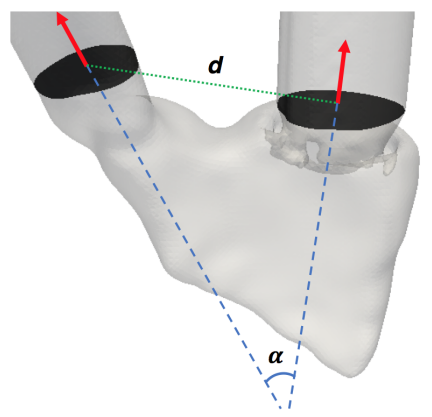

(b) Geometrical markers.

FI G URE 2 (a) Trans-tricuspid E-A wave parameters (blue) and the 6 analysed time instants (red). (b) The definition of the angle $(\alpha)$ and distance $(d)$ between the tricuspid and pulmonary valve planes.

compared. The WSS difference ( $W S S_{\text {diff }}$ ) was then calculated as the difference between the smoothed and detailed WSS medians $\left(W S S_{s}-W S S_{d}\right.$ ). WSS magnitude on the ventricular walls was analysed in both constant and transient inflow simulations.

Linear regression was used to analyse the correlation of both geometrical markers ( $\alpha$ and $d$ ) and the percentages of trabeculae with the $\Delta P_{\text {diff }}$ and also the correlation between the percentages of trabeculae and the $W S S_{\text {diff }}$. The correlation coefficient $r$ was computed for every case.

Points of interest. Four points of interest were selected inside each ventricular cavity, as shown in Fig. 1, to record blood flow velocity values at each time step and to analyse their auto-correlations in time. The four points were positioned at locations where we postulated the blood flow may have more complex and interesting behaviours: right behind the tricuspid valve (1), in the distal apex (2), at the mid-papillary cross section (3), and within the pulmonary root (4). The auto-correlation was used to analyse the degree of similarity of the velocity signal with a delayed version of itself. This function was then used to investigate whether the flow is turbulent, shown by a poor autocorrelation of the velocity in time.

Vortex formation. Coherent structures inside the ventricular cavities were studied in both steady and transient inflow simulations, using the Q-criterion [4, 12]. The applied thresholds for vortex visualisation were $9000 \mathrm{~s}^{-2}$ and 2000 $\mathrm{s}^{-2}$ for steady and transient inflow simulations respectively. Paraview was used to carry out the vortex quantification by integrating the vortices contours and obtain their total surface areas. 
TAB LE 1 Model description and constant inflow simulation results

\begin{tabular}{|c|c|c|c|c|c|c|c|c|}
\hline \multirow{2}{*}{$\begin{array}{l}\text { Hearts } \\
\text { Geometry }\end{array}$} & \multicolumn{2}{|c|}{$F_{1}$} & \multicolumn{2}{|c|}{$F_{2}$} & \multicolumn{2}{|c|}{$M_{1}$} & \multicolumn{2}{|c|}{$M_{2}$} \\
\hline & Smoothed & Detailed & Smoothed & Detailed & Smoothed & Detailed & Smoothed & Detailed \\
\hline Area inlet $\left[\mathrm{m}^{2}\right]$ & \multicolumn{2}{|c|}{$9.2 \cdot 10^{-4}$} & \multicolumn{2}{|c|}{$10.2 \cdot 10^{-4}$} & \multicolumn{2}{|c|}{$7.8 \cdot 10^{-4}$} & \multicolumn{2}{|c|}{$5.8 \cdot 10^{-4}$} \\
\hline Area outlet $\left[\mathrm{m}^{2}\right]$ & \multicolumn{2}{|c|}{$3.6 \cdot 10^{-4}$} & \multicolumn{2}{|c|}{$3.2 \cdot 10^{-4}$} & \multicolumn{2}{|c|}{$5.8 \cdot 10^{-4}$} & \multicolumn{2}{|c|}{$4.4 \cdot 10^{-4}$} \\
\hline$\alpha$ [degrees] & \multicolumn{2}{|c|}{24.8} & \multicolumn{2}{|c|}{43.9} & \multicolumn{2}{|c|}{56.2} & \multicolumn{2}{|c|}{44.7} \\
\hline$d[\mathrm{~m}]$ & \multicolumn{2}{|c|}{$6.7 \cdot 10^{-2}$} & \multicolumn{2}{|c|}{$6.7 \cdot 10^{-2}$} & \multicolumn{2}{|c|}{$6.6 \cdot 10^{-2}$} & \multicolumn{2}{|c|}{$7.2 \cdot 10^{-2}$} \\
\hline Volume $\left[\mathrm{m}^{3}\right]$ & $130.5 \cdot 10^{-6}$ & $113 \cdot 10^{-6}$ & $183.4 \cdot 10^{-6}$ & $162.5 \cdot 10^{-6}$ & $200.3 \cdot 10^{-6}$ & $164.4 \cdot 10^{-6}$ & $165.7 \cdot 10^{-6}$ & $122.1 \cdot 10^{-6}$ \\
\hline Trabeculae [\%] & \multicolumn{2}{|c|}{13.4} & \multicolumn{2}{|c|}{11.4} & \multicolumn{2}{|c|}{17.9} & \multicolumn{2}{|c|}{26.3} \\
\hline Volumetric mesh elements & $6,074,728$ & $5,395,335$ & $6,877,088$ & $4,904,622$ & $4,363,272$ & $3,547,518$ & $4,613,648$ & $3,710,093$ \\
\hline Volumetric mesh points & $1,084,088$ & 999,693 & $1,222,888$ & 825,587 & 754,418 & 663,631 & 781,814 & 699,436 \\
\hline Reynolds number at inlet & \multicolumn{2}{|c|}{3946.3} & \multicolumn{2}{|c|}{4142.4} & \multicolumn{2}{|c|}{3630} & \multicolumn{2}{|c|}{3120} \\
\hline$\Delta \mathrm{P}[\mathrm{Pa}]$ & $1125.9 \pm 22.7$ & $1195.3 \pm 29.2$ & $1170.7 \pm 96.4$ & $1280.6 \pm 36$ & $518 \pm 9.1$ & $813.7 \pm 27.6$ & $810.3 \pm 13.4$ & $979.8 \pm 27.8$ \\
\hline$\Delta \mathrm{P}_{\text {diff }}[\mathrm{Pa}]$ & \multicolumn{2}{|c|}{69.4} & \multicolumn{2}{|c|}{109.9} & \multicolumn{2}{|c|}{295.7} & \multicolumn{2}{|c|}{169.5} \\
\hline WSS maximum [Pa] & 86.39 & 31.99 & 105.82 & 29.95 & 71.69 & 30.72 & 82.14 & 36.00 \\
\hline WSS median [Pa] & 5.2 & 1.4 & 8.6 & 1.2 & 4.9 & 0.7 & 5.9 & 1.2 \\
\hline $\mathrm{WSS}_{\text {diff }}[\mathrm{Pa}]$ & \multicolumn{2}{|c|}{3.8} & \multicolumn{2}{|c|}{7.4} & \multicolumn{2}{|c|}{4.2} & & \\
\hline Vortices surface area $\left[\mathrm{m}^{2}\right]$ & $12.1 \cdot 10^{-3}$ & $105.7 \cdot 10^{-3}$ & $10.2 \cdot 10^{-3}$ & $68.3 \cdot 10^{-3}$ & $5.6 \cdot 10^{-3}$ & $83.2 \cdot 10^{-3}$ & $5.9 \cdot 10^{-3}$ & $56.3 \cdot 10^{-3}$ \\
\hline
\end{tabular}

$\triangle \mathrm{P}$ : intra-ventricular pressure drop from inlet to outlet, calculated from the mode of the pressure histograms

$\Delta P_{\text {diff }}=\Delta P_{d}-\Delta P_{s}:$ difference between detailed and smoothed pressure drops

WSS $_{\text {diff }}=$ WSS $_{\mathrm{s}}-$ WSS $_{d}$ : difference between smoothed and detailed WSS median

\section{3 | RESULTS}

The geometrical markers, model details and results of each model under constant inflow are shown in Table 1. In all of the ventricles, the intra-ventricular pressure drops were higher in the detailed geometries, with an average increase $\Delta P_{\text {diff }}$ of approximately 161.1 Pa. The magnitudes of the WSS are shown in Fig. 3. The WSS median decreased in the detailed geometries for all RVs. The median WSS of all cases can be observed in Table 1. It was observed that in all cases the maximum values of WSS occur within the smoothed geometries. The coefficients $r$ were calculated in order to quantify the correlations between $\Delta P_{\text {diff }}$ and the angles $\alpha$, the distances $d$, the percentages of trabeculae and also the correlations between $W S S_{\text {diff }}$ and the percentages of trabeculae. The four linear regression plots along with the corresponding correlation coefficient $r$ are shown in Fig. 4. The correlation coefficient $r$ shows strong correlations $(r \geq 0.7)$ between the $\Delta P_{\text {diff }}$ and $\alpha$ and weak correlations $(r<0.7)$ between the percentages of trabeculae and $\Delta P_{\text {diff }}$. The WSS median was poorly, but inversely correlated to the percentages of trabeculae $(r=-0.39)$.

The flow velocity signals at the four points of interest for smoothed and detailed $M_{2}$ RVs are shown in Fig. 12-15 of the electronic supplementary materials. The continuous time auto-correlation functions $R_{u u}$ of the flow velocity fluctuation signals $u^{\prime}(t)$ were calculated for each one of the four points of interest for both smoothed and detailed RVs. The example of $\mathrm{M}_{2} \mathrm{RV}$ is shown in Fig. 5, where $R_{u u}$ was plotted against time lag. From this figure, it can be observed that in none of the four points of the smoothed RVs $R_{u u}$ crossed the 0 value, while in the detailed cases $R_{u u}$ were 0 at least crossed once for each point of interest. Typically, laminar flows present $R_{u u}$ where the signal correlation is never lost, since symmetries in the flow were present. However, the perturbations triggered by the trabeculae induced the flows to decorrelate, which means that the blood flow velocities were transitional, going towards turbulent.

Coherent structures calculated for the constant inflow simulations are shown in Fig. 6. Q-criterion was thresholded at $9000 \mathrm{~s}^{-2}$ and coloured according to the velocity magnitude $[\mathrm{m} / \mathrm{s}]$. The results from the vortex surface area quantification can be observed in Table 2. Vortices in the smoothed RVs were fewer and tighter (with a mean vortex surface area of $8.4 \pm 3.2 \cdot 10^{-3} \mathrm{~m}^{2}$ ), while in the detailed geometries there were disruptions of the larger vortical structures and the formations of multiple small scale vortices (with a mean vortex area of $78.4 \pm 21.3 \cdot 10^{-3} \mathrm{~m}^{2}$ ).

The six time instants, selected from the E-A wave trans-tricuspid inflow functions, are shown in Fig. 2(a) along with 

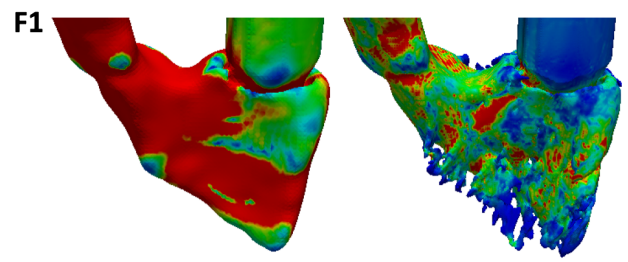

M1
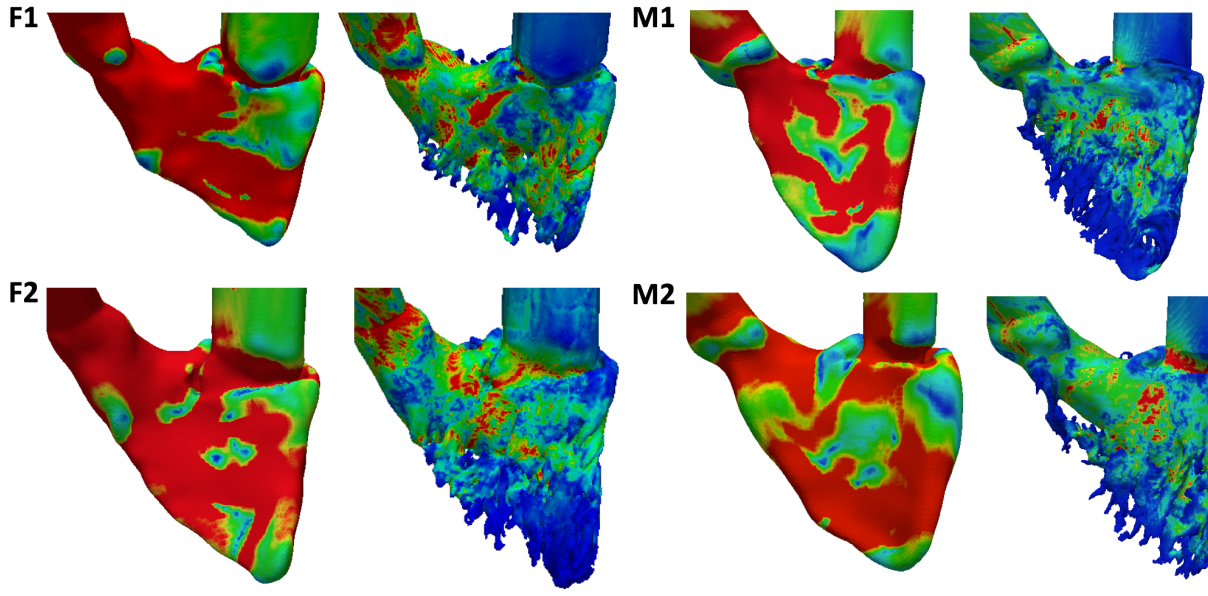

M2
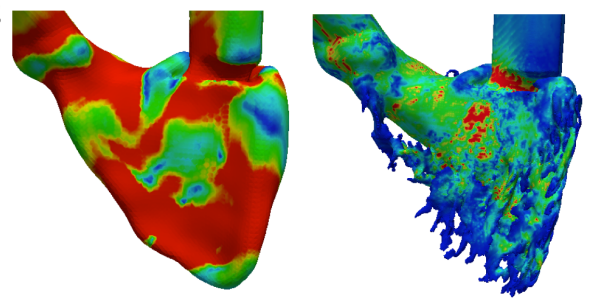

WSS (Pa)

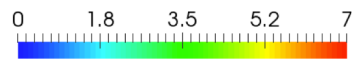

FIGURE 3 Magnitudes of the WSS during constant inflow simulations in RVs with smoothed and detailed geometry.

TAB LE 2 Total vortex surface area $\left[\mathrm{m}^{2}\right]$ for the six instants (1-6) of the synthetic E-A wave in subject $F_{1}$.

\begin{tabular}{lcccccc}
\hline Geometry & $\mathbf{1}$ & $\mathbf{2}$ & $\mathbf{3}$ & $\mathbf{4}$ & $\mathbf{5}$ & $\mathbf{6}$ \\
\hline Smoothed & $3.7 \cdot 10^{-3}$ & $13.2 \cdot 10^{-3}$ & $16.8 \cdot 10^{-3}$ & $2.5 \cdot 10^{-3}$ & $1.7 \cdot 10^{-3}$ & $0.2 \cdot 10^{-3}$ \\
Detailed & $10.9 \cdot 10^{-3}$ & $71.5 \cdot 10^{-3}$ & $124.8 \cdot 10^{-3}$ & $58.7 \cdot 10^{-3}$ & $37.3 \cdot 10^{-3}$ & $26.7 \cdot 10^{-3}$ \\
\hline
\end{tabular}

the inflow wave. An example of the vortex analysis at those time instants, is shown in Fig. 7, for subject $F_{1}$. In brief, early E wave (1), E wave peak (2), late E wave (3), early A wave (4), A wave peak (5) and late A wave (6) were shown. In the smoothed ventricles, vortices were few and the generation of the vortex rings were clearly visible during the E wave. On the other hand, in the detailed cases, the vortex rings were disrupted and there were the generations of multiple secondary vortices. For each time instant, vortices were quantitatively compared and are shown in Table 2 . The total surfaces of the vortices in $F_{1}$ were larger in the detailed compared to the smoothed geometries. Fig. 7 shows the vortices for subject $F_{1}$. The maximum numbers of vortices occurred in both geometries at the downfalls of the $E$ wave (time instant 3 ) and during the rises of the inlet velocities of the $A$ wave (time instant 4).

Additionally, WSS in peak $E$ and peak A waves were analysed and compared between the smoothed and detailed geometries. An example of the $F_{1}$ RV case is shown in Fig. 8; here, WSS was higher in the smoothed compared to the detailed case, for both peak $E$ and $A$ waves.

\section{4 | DISCUSSION}

To our knowledge, this is the first time that detailed endocardial structures have been considered in haemodynamic simulations of the RV. Here, the reconstructed trabeculations had minimum cross-sectional areas of $1 \mathrm{~mm}^{2}$. The results obtained from these characterizations of the RV geometries indicate that the blood flows within this chamber are much 
more complex than the previous state of the art simulations have assumes. In other words, the presence of detailed structures increases the intra-ventricular pressure drops with a mean $\Delta P_{\text {diff }}$ of $161.1 \pm 98.7 \mathrm{~Pa}$. It was also observed that the existences of trabeculae, PMs and moderator bands increases the vorticities of the blood flows within these chambers, while the WSS decreases, with a mean $W S S_{\text {diff }}$ of $5 \pm 1.6 \mathrm{~Pa}$. Furthermore, under the flow regime of the presented models, it was clear that blood flow patterns within the detailed geometries tends to be more turbulent, presenting complex coherent flow structures.

Intra-ventricular pressure drop is a parameter which we considered to have an important role in analysing ventricular functions and is commonly used clinically as markers for cardiac pathologies such as ventricular ischemia [22] or valve stenosis [24]. We hypothesise here, that a fraction of that intra-ventricular pressure drop could arise from the dissipations of energies due to the coarseness of a given ventricular wall.

Within our small sample size, it was possible to observe a slight gender dissimilarity regarding the distributions of trabeculae (see Table 1); Lower $\Delta P_{\text {diff }}$ between the detailed and smoothed ventricles were found for the female RVs. It is interesting that the intra-ventricular pressure drops were highly correlated to the angles between the inlet and outlet valves, but only weakly correlated to the percentages of the volumes occupied by the endocardial structures in these models. The male geometries elicited higher angles $\alpha$ between the valve rings and higher percentages of trabeculae.

In all of the RVs, the presence of both trabeculae and PMs were associated with decreased WSS. There was a weak negative correlation $(r=-0.39$ ) between the amounts of the WSS decreases and the percentages of trabecular volume. It is interesting to note, that in the smoothed geometries the mode WSS ranges between $0.15-3.7 \mathrm{~Pa}$, while in detailed meshes the mode dropped to $0.05-0.1 \mathrm{~Pa}$ (see Fig. 8-11 of the supplementary materials). In future studies, it may be of interest to examine whether the distributions of endocardial structures would be correlated to reductions of WSS within a specific range, but many more subjects would be required to do so. It should also be noted, that Wiputra et al. [36] suggested that the high vorticity regions were related to a higher WSS, through the generation of forces which they determined to be tangential to the ventricular walls. These relationships were observable also in our smoothed RV simulations, when comparing results in Fig. 3,6 (constant inflow) and 7,8 (transient inflow): higher WSSs were indeed found in the vicinities of larger vortices.

Vortex analysis also demonstrated that the presence of detailed endocardial structures disrupted the main vortex ring in both constant and transient inflow simulations, generating small scale secondary vortices. Vortices in smoothed $\mathrm{RVs}$ were fewer and more compact and these results were in accordance with the few larger, tighter ones observed for the simplified RV models of Vruddhula et al. [35], Wiputra et al. [36], and from the large scale vortical motions observed by Pasipoularides et al. [20].

The velocities at the selected points within both smoothed and detailed geometries indicated that the simulations did not reach steady flow states, even though the overall pressure distributions at the inlet and outlet did not vary within the last derived ten time frames (approximately $50 \mathrm{~ms}$ of simulations) in most of these models: except for the detailed case of subject $F_{2}$ (distributions available in Fig. 16 of the supplementary material). We hypothesise that these pressure variations arose from the reflections created by the increased numbers of endocardial structures that impede the laminar flows of blood (velocity vectors at the inlet of both the smoothed and detailed cases $F_{2}$ can be observed in Fig. 17-18 of the supplementary material). This was important because within our models, the pre-loads of the RVs were affected by the endocardial structures. 


\section{5 | LIMITATIONS}

The main limitation of our present work, is the use of rigid walls, without motions or FSI. Given this limitation, we cannot provide patient-specific, physiologically relevant information as to overall function. RV walls motion was included in earlier works like the one of Su et al. [28]. RV geometry was reconstructed at end of diastole, interpolation was applied to increase MRI temporal resolution and then a smooth deformation following the whole cardiac cycle was applied to the ventricular walls. The authors showed how motion of the RV walls plays an important role in simulations results like pressure drop and vorticity. Moreover, previous works on LV detailed models have included moving walls by increasing and decreasing the ventricular volumes linearly. Yet, this is not directly applicable to the RV haemodynamics, since the pumping effects of the RV arises mainly from a basal plane descent, during a given heart beat; which does not correspond to a linear scaling of the geometry. However, the computational costs and methods to create an FSI simulation of a geometry like the ones we present, are yet to be reported. However, although we did not include FSI, the results of our CFD simulations allows one to elucidate for the first time, the relative effects of RV endocardial structures on the blood flow; shedding light on RV trabecular function. Specifically, we were able to show the impacts of ventricular trabeculae on associated blood flows. Yet, these effects on blood flows were minimal when analysing the haemodynamics in smoothed RV models. Therefore, we believe that future studies, should include these detailed structures for RV haemodynamic computational modelling. We consider here that another important limitation was our utilization of rigid, fully open valve leaflets: in future studies the incorporation of valve motions could help to better understand the underlying behaviours of three-dimensional intra-cardiac flows [27]. Thus, including valve motions along with the motions of the ventricular walls is an ongoing mission of our future work. The last limitation we should note, as state above was the low number of subjects analyzed in this study: with a larger subject sampling our findings regarding gender differences could potentially provide more statistically significant results.

\section{6 | CONCLUSIONS}

Here we presented CFD simulations of RV models with high geometrical details which, to the best of our knowledge, has not been described yet in the blood flow simulation literature. Moreover, this is the first time that intra-ventricular pressure drops were analysed as means to investigate the effects of trabeculae and/or PMs on RV haemodynamic modelling. Our study demonstrates that detailed endocardial structures have significant influences on the RV blood flow dynamics by increasing the intra-ventricular pressure drops, decreasing the WSS and increasing the vortex formations. Hence, RV haemodynamics simulations with smoothed endocardial surfaces do not describe the full complexities of blood flow within the RV chambers. Moreover, the velocity auto-correlation analysis indicated how the presence of detailed endocardial structures generated transitional turbulent flow, which was absent in the smoothed geometries, within our simulation flow regime. Finally, gender dissimilarities were identified, but future studies are need with larger population.

\section{ACKNOWLEDGMENTS}

The DICOM datasets were provided by the Visible Heart ${ }^{\circledR}$ Laboratory, obtained by MRI scanning of perfusion fixed hearts that were graciously donated by the organ donors and their families through LifeSource.

Part of the simulation hours were provided by the CompBioMed project in the Archer supercomputer, EPCC, UK. 


\section{CONFLICT OF INTEREST}

The authors have no conflict of interest to disclose.

\section{SUPPORTING INFORMATION}

The following supporting information is available as part of the on-line article:

Table 1. Heart database: human characteristics and medical histories

Figure 1. High resolution MRI datasets of the four human hearts, short axis view, mid-cavity slice.

Figure 2. $F_{1,2}$ and $M_{1,2}$ RV myocardial surfaces: smoothed (left) and detailed (right) tetrahedral wire-frame meshes. Shown are transverse slices (top) and inner views from right below the tricuspid valve (bottom) for each smoothed/detailed pair. The actual sizes of the zoomed region are reported.

Figure 3. RV labels for inflow, outflow and walls boundary conditions.

Figure 4. Normalised pressure histograms of smoothed and detailed $F_{1} R V$ inlet and outlet volumes at the last time instant of constant inflow simulation.

Figure 5. Normalised pressure histograms of smoothed and detailed $F_{2} R V$ inlet and outlet volumes at the last time instant of constant inflow simulation.

Figure 6. Normalised pressure histograms of smoothed and detailed $M_{1} R V$ inlet and outlet volumes at the last time instant of constant inflow simulation.

Figure 7. Normalised pressure histograms of smoothed and detailed $M_{2} R V$ inlet and outlet volumes at the last time instant of constant inflow simulation.

Figure 8. Normalised WSS histograms of smoothed and detailed $F_{1} R V$ at the last time instant of constant inflow simulation. Median WSS values are indicated with dashed lines. The range $0-30 \mathrm{~Pa}$ is highlighted. Maximum smoothed and detailed WSS are reported in the top-right corner. The presence of endocardial detail reduces the shear stress on the ventricular walls.

Figure 9. Normalised WSS histograms of smoothed and detailed $F_{2} R V$ at the last time instant of constant inflow simulation. Median WSS values are indicated with dashed lines. The range $0-30 \mathrm{~Pa}$ is highlighted. Maximum smoothed and detailed WSS are reported in the top-right corner. The presence of endocardial detail reduces the shear stress on the ventricular walls.

Figure 10. Normalised WSS histograms of smoothed and detailed $M_{1} R V$ at the last time instant of constant inflow simulation. Median WSS values are indicated with dashed lines. The range $0-30 \mathrm{~Pa}$ is highlighted. Maximum smoothed and detailed WSS are reported in the top-right corner. The presence of endocardial detail reduces the shear stress on the ventricular walls.

Figure 11. Normalised WSS histograms of smoothed and detailed $M_{2} R V$ at the last time instant of constant inflow simulation. Median WSS values are indicated with dashed lines. The range $0-30 \mathrm{~Pa}$ is highlighted. Maximum smoothed and detailed WSS are reported in the top-right corner. The presence of endocardial detail reduces the shear stress on the ventricular walls.

Figure 12. Point of interest 1 for smoothed and detailed $M_{2}$ RV. Shown is the recorded velocity $u(t)$; constant inflow simulations. The velocity signal is transient in both smoothed and detailed RV.

Figure 13. Point of interest 2 for smoothed and detailed $M_{2}$ RV. Shown is the recorded velocity $u(t)$; constant inflow simulations. The velocity signal is transient in both smoothed and detailed RV.

Figure 14. Point of interest 3 for smoothed and detailed $M_{2}$ RV. Shown is the recorded velocity $u(t)$; constant inflow simulations. The velocity signal is transient in both smoothed and detailed RV. 
Figure 15. Point of interest 4 for smoothed and detailed $M_{2}$ RV. Shown is the recorded velocity $u(t)$; constant inflow simulations. The velocity signal is transient in both smoothed and detailed RV.

Figure 16. Normalised pressure histograms of smoothed and detailed $F_{2} R V$ inlet and outlet volumes for the last 10 time instants of constant inflow simulation. Pressure oscillation is noticeable for the detailed case.

Figure 17. Inlet velocity glyph representation for the smoothed $F_{2} R V$ in constant inflow simulation. Glyph are coloured by the pressure. As can be seen by the velocity vectors, blood flow reflection is less compared to the detailed case.

Figure 18. Inlet velocity glyph representation for the detailed $F_{2} R V$ in constant inflow simulation. Glyph are coloured by the pressure. Here, as can be seen by the velocity vectors, there is a higher blood flow reflection due to the presence of detailed endocardial structures right below the valve.

\section{REFERENCES}

[1] Attene M, Falcidieno B. ReMESH: An Interactive Environment to Edit and Repair Triangle Meshes. IEEE International Conference on Shape Modeling and Applications 2006;p. 41-41. DOI:10.1109/SMI.2006.29.

[2] Bazilevs Y, Gohean JR, Hughes ea. Patient-specific isogeometric fluid-structure interaction analysis of thoracic aortic blood flow due to implantation of the Jarvik 2000 left ventricular assist device. Comput Methods in Appl Mech Eng 2009;198(45-46):3534-3550. DOI:10.1016/j.cma.2009.04.015.

[3] Capuano F, Coppola G, Rández L, de Luca L. Explicit Runge-Kutta schemes for incompressible flow with improved energyconservation properties. Journal of Computational Physics 2017;328:86-94.

[4] Chakraborty P, Balachandar S, Adrian RJ. On the relationships between local vortex identification schemes. J Fluid Mech 2005;535:189-214. DOI:10.1017/S0022112005004726.

[5] Charnyi S, Heister T, Olshanskii MA, Rebholz LG. On conservation laws of NavierĐStokes Galerkin discretizations. Journal of Computational Physics 2017;337:289 - 308.

[6] CIBC. Seg3D: Volumetric Image Segmentation and Visualization. Scientific Computing and Imaging Institute 2016;.

[7] Efron B, Tibshirani R. Bootstrap Methods for Standard Errors, Confidence Intervals, and Other Measures of Statistical Accurancy. Statistical Science 1986;1(1):54-77.

[8] Ghosh E, Caruthers SD, Kovács SJ. E-wave generated intraventricular diastolic vortex to L-wave relation: model-based prediction with in vivo validation. J Appl Physiol 2014;117(3):316-324. DOI:10.1152/japplphysiol.00215.2014.

[9] Haddad F, Hunt SA, Rosenthal DN, Murphy DJ. Right ventricular function in cardiovascular disease, part I: Anatomy, physiology, aging, and functional assessment of the right ventricle. Circulation 2008;117(11):1436-1448. DOI:10.1161/CIRCULATIONAHA.107.653576.

[10] Henderson A. ParaView Guide, A Parallel Visualization Application. Kitware Inc 2007;.

[11] Houzeaux G, Vázquez M, Aubry R, Cela JM. A massively parallel fractional step solver for incompressible flows. J Comput Phys 2009;228(17):6316-6332. DOI:10.1016/j.jcp.2009.05.019.

[12] Hunt JCR, Wray AA, Moin P. Eddies, streams, and convergence zones in turbulent flows. CTR Summer Program Proceeding 1988;(1970):193-208.

[13] Jofre L, Lehmkuhl O, Ventosa J, Trias FX, Oliva A. Conservation Properties of Unstructured Finite-Volume Mesh Schemes for the Navier-Stokes Equations. Num Heat Tran, Part B: Fund 2013;65:53-79. DOI:10.1080/10407790.2013.836335.

[14] Koullapis P, Kassinos SC, Muela J, Perez-segarra C, Rigola J, Lehmkuhl O, et al. Regional aerosol deposition in the human airways : The SimInhale benchmark case and a critical assessment of in silico methods. European Journal of Pharmaceutical Sciences 2017;(In Press). 
[15] Kulp S, Gao M, Zhang S, et al. Using high resolution cardiac CT data to model and visualize patient-specific interactions between trabeculae and blood flow. LNCS 2011;6891(PART 1):468-475. DOI:10.1007/978-3-642-23623-5_59.

[16] Labelle F, Shewchuk JR. Isosurface stuffing. ACM Transactions on Graphics 2007;26(3):57. DOI:10.1145/1276377.1276448.

[17] Lantz J, Henriksson L, Persson A, Karlsson M, Ebbers T. Patient-Specific Simulation of Cardiac Blood Flow From HighResolution CT. J Biomech Eng 2016;138(December):1-9. DOI:10.1115/1.4034652.

[18] Lee N, Taylor MD, Banerjee RK. Right ventricle-pulmonary circulation dysfunction: a review of energy-based approach. Biomedical engineering online 2015;14 Suppl 1(Suppl 1):S8. DOI:10.1186/1475-925X-14-S1-S8.

[19] Lehmkuhl O, Houzeaux G, Avila M, Owen H, Vazquez M, Mira D. A low dissipation finite element scheme for the large eddy simulation on complex geometries. 19h International Conference on Finite Elements in Flow Problems 2017;.

[20] Pasipoularides A, Shu M, Shah A, et al. Diastolic right ventricular filling vortex in normal and volume overload states. Am J Physiol Heart Circ Physiol 2003;284(4):H1064-H1072. DOI:DOI 10.1152/ajpheart.00804.2002.

[21] Pasipoularides A, Shu M, Shah A, et al. RV instantaneous intraventricular diastolic pressure and velocity distributions in normal and volume overload awake dog disease models. Am J Physiol Heart Circ Physiol 2003;285(5):H1956-H1965. DOI:10.1152/ajpheart.00373.2003.

[22] Popovic ZB, Prasad A, Garcia MJea. Relationship among diastolic intraventricular pressure gradients, relaxation, and preload: impact of age and fitness. Am J Physiol Heart Circ Physiol 2006;290(4):H1454-9. DOI:10.1152/ajpheart.00902.2005.

[23] Qi C. Maximum Entropy for Image Segmentation based on an Adaptive Particle Swarm Optimization. Appl Math Inform Sci 2014;8(6):3129-3135. DOI:10.12785/amis/080654

[24] Sabbah HN, Anbe DT, Stein PD. Negative intraventricular diastolic pressure in patients with mitral stenosis: Evidence of left ventricular diastolic suction. Am J Cardiol 2017 jul;45(3):562-566. DOI:10.1016/S0002-9149(80)80005-1.

[25] Schindelin J, Arganda-Carreras I, Frise E, Kaynig, et al. Fiji: an open-source platform for biological-image analysis. Nat Meth 2012;9(7):676-682.

[26] Schmidt R, Singh K. Meshmixer: an interface for rapid mesh composition. ACM SIGGRAPH Talks 2010;p. 2006. DOI:10.1145/1837026.1837034.

[27] Su B, et al. Cardiac MRI based numerical modeling of left ventricular fluid dynamics with mitral valve incorporated. J of Biomech 2016;DOI:10.1016/j.jbiomech.2016.03.008.

[28] Su B, et al. Numerical Modeling of Blood Flow in Patient-Specific Right Ventricle with Pulmonary Arterial Hypertension Based on MRI. International Conference for Innovation in Biomedical Engineering and Life Sciences, IFMBE Proceedings 56 2016;.

[29] Trias FX, Lehmkuhl O. A Self-Adaptive Strategy for the Time Integration of Navier-Stokes Equations. Numerical Heat Transfer, Part B: Fundamentals 2011;60(2):116-134.

[30] Trias FX, Lehmkuhl O, Oliva A, Pérez-Segarra CD, Verstappen RWCP. Symmetry preserving discretisation of Navier-Stokes equations on collocated unstructured grids. J of Comput Physics 2014;258:246-267. DOI:10.1016/j.jcp.2013.10.031.

[31] Vazquez M, Houzeaux G, Koric S, Artigues A, Aguado-Sierra J, Arís R, et al. Alya: Multiphysics engineering simulation toward exascale. J of Comput Science 2016;14(Supplement C):15 - 27. The Route to Exascale: Novel Mathematical Methods, Scalable Algorithms and Computational Science Skills. 
[32] Vedula V, Seo JH, Lardo AC, Mittal R. Effect of trabeculae and papillary muscles on the hemodynamics of the left ventricle. Theor Comp Fluid Dyn 2016;30(1-2):3-21. DOI:10.1007/s00162-015-0349-6.

[33] Verstappen RWCP, Veldman AEP. Symmetry-preserving discretization of turbulent flow. J Comput Phys 2003;187:343368. DOI:10.1016/j.jbiomech.2017.06.046.

[34] Voelkel NF, Quaife RA, Leinwand LA, Barst RJ, McGoon MD, Meldrum DR, et al. Right Ventricular Function and Failure. Circulation 2006;114(17):1883-1891. DOI:10.1161/CIRCULATIONAHA.106.632208.

[35] Vruddhula V, Borazjani I. 3D Flow Simulations In A Simplified Right Ventricle Model. The American Society of Mechanical Engineering 2016;p. 1-9.

[36] Wiputra H, Quan Lai C, Ling Lim G, et al. Fluid Mechanics of Human Fetal Right Ventricles from Image-Based Computational Fluid Dynamics Using 4D Clinical Ultrasound Scans. Am J Physiol Heart Circ Physiol 2016;311:H1498-H1508. DOI:10.1152/ajpheart.00400.2016.

[37] Yang C, Tang D, I H, et al. In vivo MRI-Based 3D FSI RV/LV Models for Human Right Ventricle and Patch Design for Potential Computer-Aided Surgery Optimization. Comput Struct 2007;85:988-997. DOI:10.1016/j.compstruc.2006.11.008. 

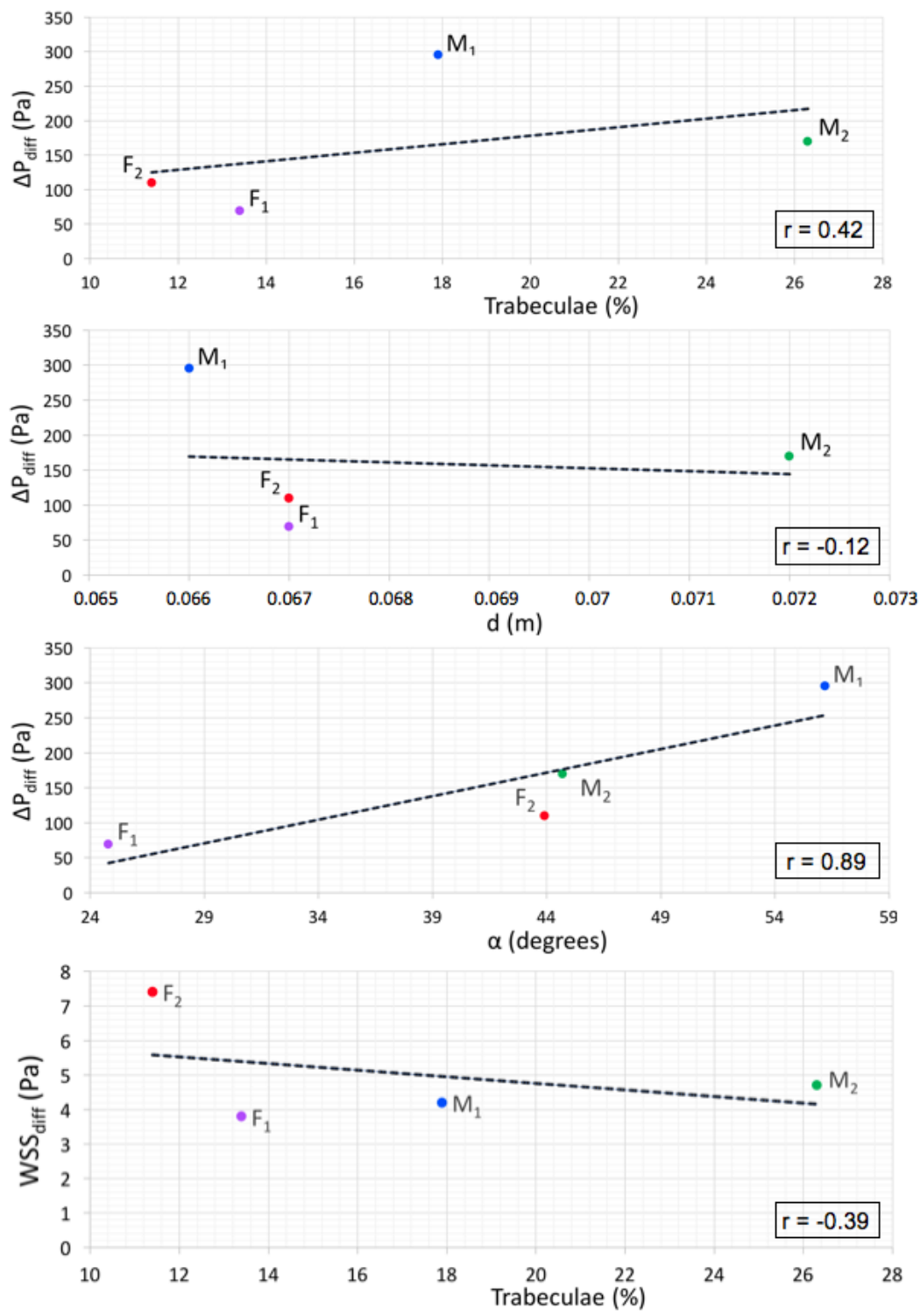

FIGURE 4 Constant inflow simulations: linear regression plots of $\Delta P_{\text {diff }}$ versus the angle $\alpha$, the distance $d$, the percentage of trabeculae along with the regression plot of $W S S_{\text {diff }}$ against the percentage of trabeculae. The correlation coefficient $r$ is indicated at the bottom-right corner of each graph. 

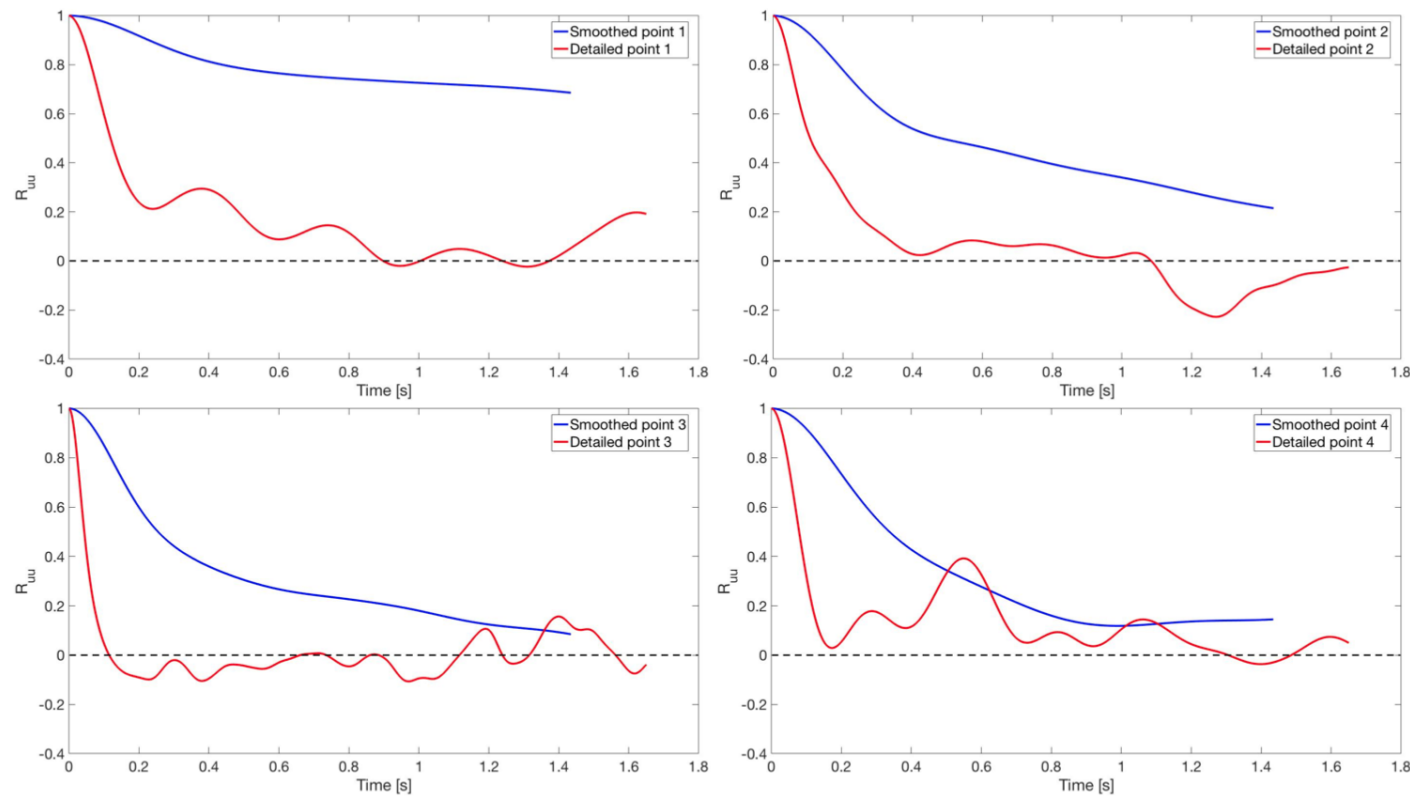

FIGURE 5 Auto-correlation of flow velocity for the points of interest $(1-4)$ for smoothed (blue) and detailed (red) $\mathrm{M}_{2} \mathrm{RV}$. Shown is the auto-correlation function $R_{u u}$ of the velocity $u(t)$; constant inflow simulations.

F1

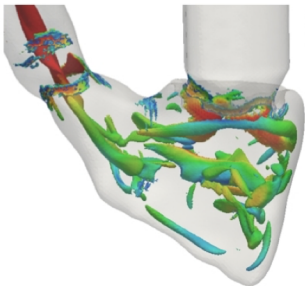

F2

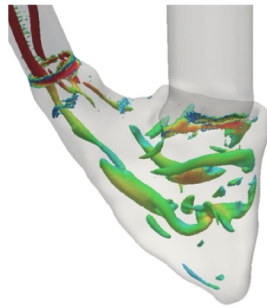

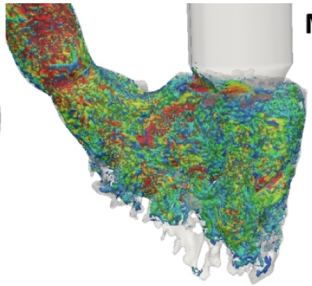

M1
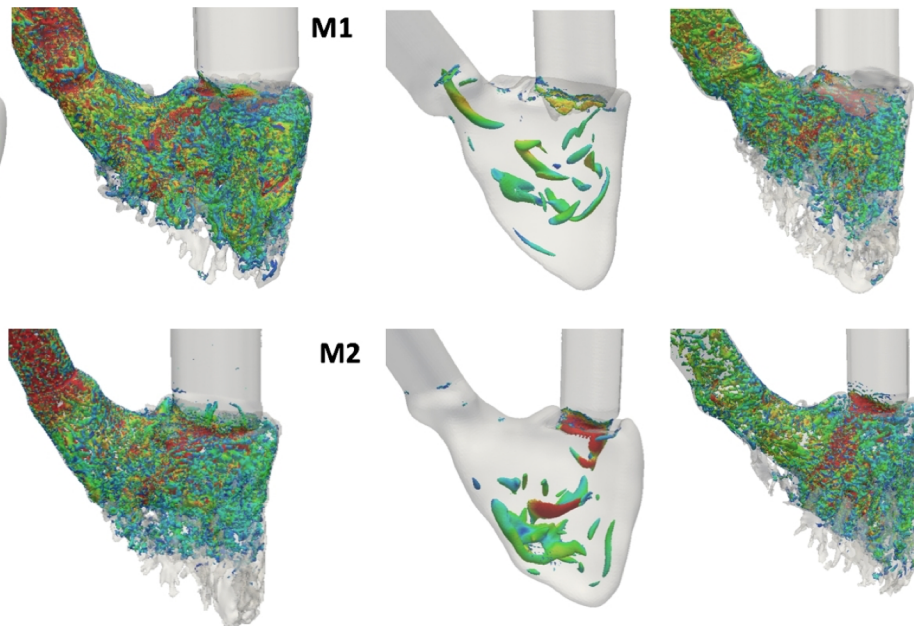

M2
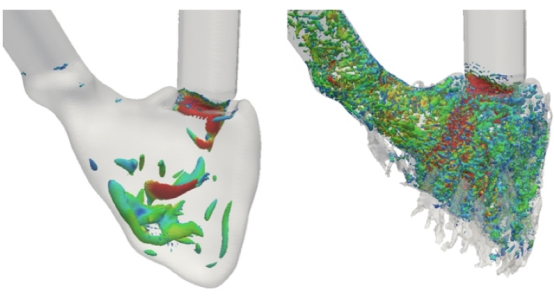

Velocity Magnitude $(\mathrm{m} / \mathrm{s})$

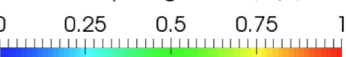

F I GURE 6 Vortex quantification using the Q-criterion thresholded at $9000 \mathrm{~s}^{-2}$ of constant inflow simulations in all RVs with smoothed and detailed geometry. Vortices are coloured by the velocity magnitude $[\mathrm{m} / \mathrm{s}]$. 
1

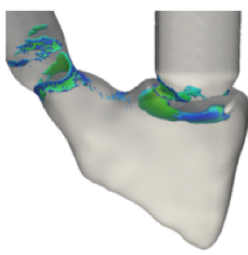

2

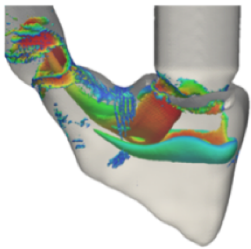

3

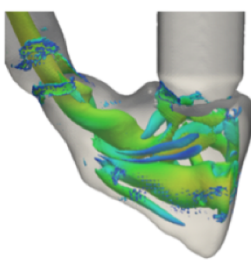

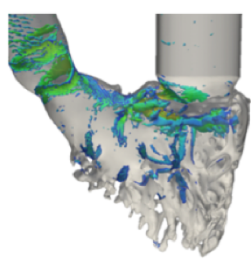
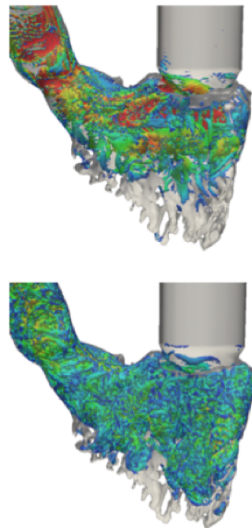

4

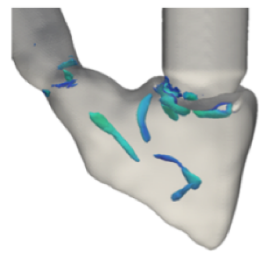

5



6

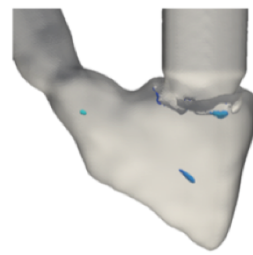

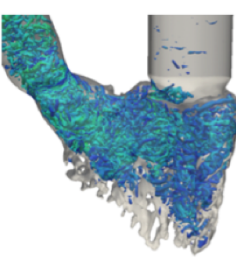
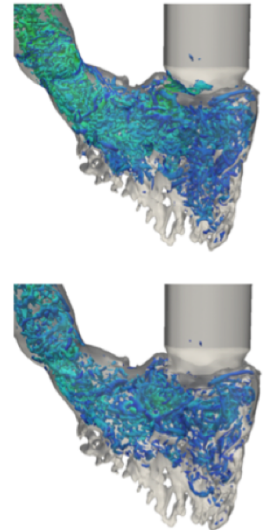

Velocity Magnitude (m/s)

0 0.25 0.5 0.75 1

FIGURE 7 Comparison of the vortices in E-A wave inflow simulations within smoothed and detailed subject $F_{1}$. Shown is the Q-criterion thresholded at $2000 \mathrm{~s}^{-2}$. Vortices are coloured by the velocity magnitude [m/s]. 

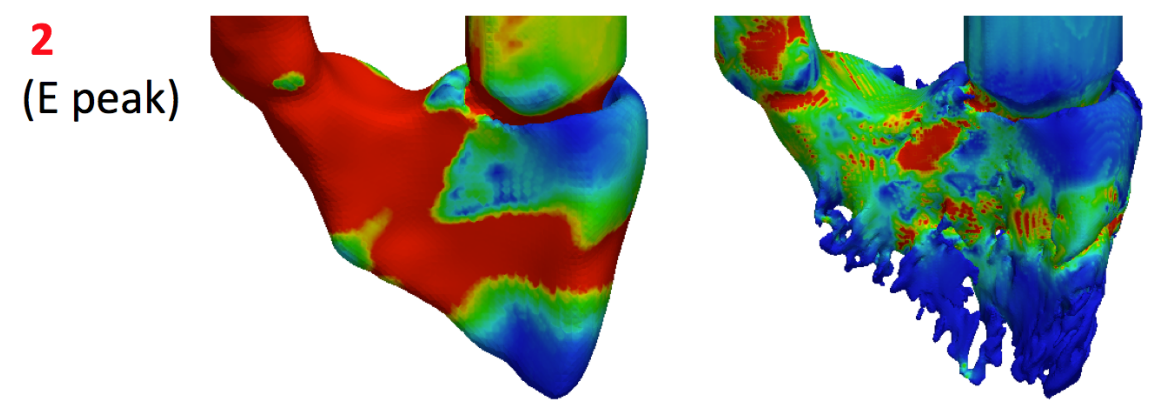

5
(A peak)
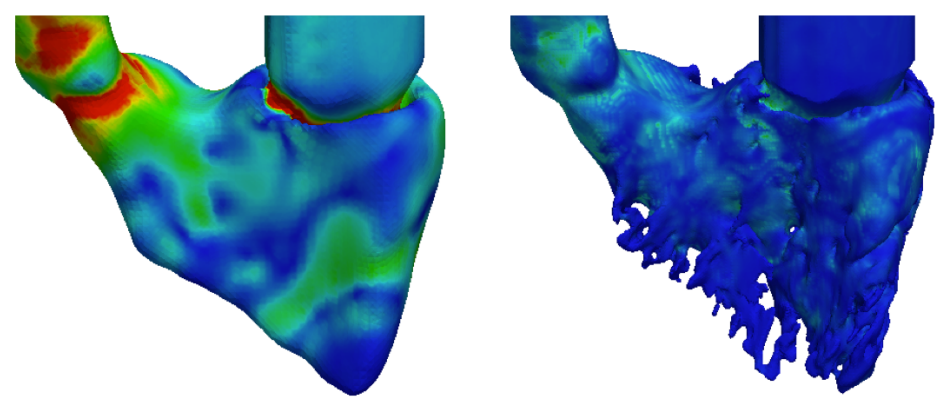

WSS (Pa)

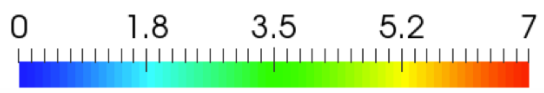

FIGURE 8 Magnitude of the WSS during peak E (time instant 2) and peak A (time instant 5) in smoothed and detailed $F_{1} R V$. 